\title{
Phase transition in current-biased arrays of small Josephson junctions
}

\author{
C. D. Porter and D. Stroud \\ Department of Physics, The Ohio State University, Columbus, Ohio 43210, USA
}

(Received 12 June 2010; published 2 November 2010)

\begin{abstract}
We present a variational approach to treat the metastable superconducting state in an array of small Josephson junctions driven by an applied current. Using this approach, we calculate an approximate phase diagram of such an array with a current bias. Our approach is a generalization of one previously used for such an array at zero applied current. We find that, for a given array, a superconducting to nonsuperconducting transition at zero temperature can be achieved either by varying the magnitude of the applied current at fixed direction or by varying the direction of the applied current at fixed magnitude. We examine this transition in two-dimensional arrays on both square and triangular lattices, and in a simple cubic array. We also calculate the dependence of this transition on the direction of the applied current. For a given array and bias current, a superconductor-toinsulator transition also takes place as the temperature is increased. Using the variational approach, we calculate critical values of the junction parameters, temperature, and current for this transition on three different lattices.
\end{abstract}

DOI: 10.1103/PhysRevB.82.184503

PACS number(s): 74.81.Fa, 74.50.+r, 74.40.Kb, 74.78.Na

\section{INTRODUCTION}

Josephson junction arrays have been extensively investigated, both experimentally and theoretically, over the past several decades. ${ }^{1}$ One topic of interest has been phase transitions in Josephson junction arrays, both with and without an applied magnetic field. These include analytical theories and quantum Monte Carlo calculations for arrays of small (underdamped) junctions (see, for example, Refs. 2-9) and large (overdamped) junctions (e.g., Refs. 10-12), and a range of experiments (see, for example, Refs. 13-18). In addition to arrays of Josephson junctions prepared from superconducting components, recent experimental work has shown that analogous phase transitions can be observed in arrays of Bose atoms, or Bose condensates, confined to optical lattices. ${ }^{19,20}$ Besides studies of phase transitions, much work has been carried out on the dynamical response of Josephson arrays, and especially the current-voltage $(I-V)$ characteristics, ${ }^{21,22}$ have been widely studied. Josephson arrays have been discussed as possible generators of coherent high-frequency (gigahertz) voltage signals ${ }^{23}$ and as superconducting quantum interference filters for use as sensitive detectors of magnetic fields. ${ }^{24}$ Josephson arrays have also been treated as possible analogs for thin, especially granular, superconducting films, ${ }^{25}$ and even for individual $\mathrm{CuO}_{2}$ layers in the high- $T_{c}$ cuprate superconductors, ${ }^{26}$ while small groups and arrays of Josephson junctions have been proposed as qubits, and even topologically protected qubits, for quantum computation. . $^{27,28}$

A Josephson array typically consists of a periodic arrangement of superconducting (S) islands in a nonsuperconducting host. If the host is a normal metal, or a superconductor with a lower transition temperature, this arrangement will form a network of overdamped superconducting-normalsuperconducting (SNS) junctions, in which capacitive (charging) energies are unimportant. If, however, the host is semiconducting or insulating, the array will be composed of superconducting-insulating-superconducting (SIS) junctions.

In the SNS case, the most important term in the array Hamiltonian is the Josephson coupling energy between two superconducting islands. The Josephson coupling term between the $i$ th and $j$ th superconducting grain generally takes the form $-\left(E_{J}\right)_{i j} \cos \left(\phi_{i}-\phi_{j}\right)$, where $\left(E_{J}\right)_{i j}$ is the Josephson energy, and $\phi_{i}$ and $\phi_{j}$ are the phases of the superconducting order parameters on grains $i$ and $j .{ }^{29}$ In this (SNS) case, the array will undergo a transition from a phase-disordered to a phase-ordered state with decreasing temperature $T$. For a $d$-dimensional array, the transition is expected to be in the same universality class as the $d$-dimensional XY model, and, in particular, will be of the Berezinskii-Kosterlitz-Thouless form in $d=2.30,31$

In an array of SIS junctions, however, the Josephson coupling energy generally competes with a charging energy related to the finite capacitance of the individual superconducting islands. If the capacitance is sufficiently small, the charging energy will prevent phase ordering at any temperature, but for grains of larger capacitance, a phase-ordering transition can still take place. At $T=0$, there is generally a critical value of the ratio $U / E_{J}$, where $U$ is the charging energy, below which the array is superconducting and above which it is insulating. This transition is considered to be a prototype of a quantum-phase transition and in $d$ dimensions the typical model Hamiltonian is in the universality class of the $(d+1)$-dimensional XY model. ${ }^{32}$

In the absence of a bias current, phase transitions in both SNS and SIS junction arrays have been extensively studied, both theoretically and experimentally, for a wide range of models and materials, both at zero and finite magnetic fields (see Ref. 1 for a review of such studies up to about 2000). The effects of thermal fluctuations are also of great importance in such arrays, both because of their effects in individual junctions ${ }^{33}$ and because of the reduced dimensionality of the arrays. ${ }^{34}$

The effects of a bias current on such phase transitions have been studied by a several groups in SNS arrays but not SIS arrays. For ordered SNS arrays, calculations and experiments have suggested that a variety of phase-transitionlike events occur at finite current, especially in the presence of an applied transverse magnetic field. Such a field typically produces a periodic distribution of vortices which can be de- 


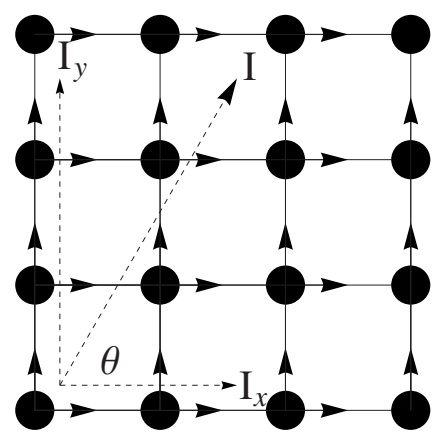

FIG. 1. Schematic of the geometry considered for a Josephson junction array on a square lattice. Currents $I_{x}=I \cos \theta$ and $I_{y}$ $=I \sin \theta$ are applied to junctions along the $x$ and $y$ axes, as indicated. The total current density is $(I \cos \theta / a) \hat{x}+(I \sin \theta / a) \hat{y}$, where $a$ is the lattice constant, and flows in the direction indicated by the arrow labeled $I$.

pinned by the applied current. ${ }^{35-44}$ When the array has even weak disorder, as well as a finite current and a finite applied magnetic field, these arrays have a rich variety of behavior, with transitions between pinned and different kinds of moving ordered or partially ordered vortex states. ${ }^{10}$ For example, Fisher et al. ${ }^{41}$ have investigated phase transitions at a field of one-half flux quantum per plaquette. In this case, the phase diagram represents regions of phase space in which a vortex lattice is either unpinned or pinned by a periodic pinning potential. The physically important case of a disordered pinning potential and a finite current has been studied, mainly numerically, by several groups. ${ }^{40,44}$ These studies all explore the phase diagram of a classical model.

In this paper, we calculate the phase diagram of a quantum model corresponding to an SIS Josephson array in the presence of an applied current. Specifically, we develop a variational treatment of phase transitions of such an array in an applied current. Our model Hamiltonian includes both Josephson and charging energies, and also a term which depends on the dc current, which is applied at an arbitrary angle with respect to the symmetry axis of the array. The main result of our approach is that, for any angle, there is a current-dependent critical ratio $U / E_{J}$ at which the array undergoes a transition from superconducting to insulating. The transition appears to be first order in our variational treatment, though this feature could change in a more exact analysis.

The remainder of this paper is organized as follows. In Sec. II, we describe our Hamiltonian and our variational method of treating it. Our numerical results are presented in Sec. III, followed by a brief discussion in Sec. IV.

\section{FORMALISM}

\section{A. Hamiltonian}

To describe the model, we first consider a square array of $N$ superconducting islands connected by Josephson junctions with an applied current at an angle $\theta$ to the $x$-axis (see Fig. 1 ). We initially specialize to the case $\theta=\pi / 4$ (current along the diagonal). In this case, the Hamiltonian is assumed to be

$$
H=\frac{U}{2} \sum_{i=1}^{N} n_{i}^{2}+E_{J} \sum_{\langle i j\rangle}\left[1-\cos \left(\phi_{i}-\phi_{j}\right)-\frac{I_{i j}}{I_{c}}\left(\phi_{i}-\phi_{j}\right)\right] .
$$

Here $U$ represents the charging energy of a single island, $n_{i}$ is the number operator representing the excess Cooper pairs on the $i$ th island. $U$ is, in general, related to the inverse of the capacitance of the $i$ th island. $\phi_{i}$ is the phase of the superconducting order parameter on the $i$ th island, $I_{c}$ is the critical current of each junction and $I_{i j}$ is the current from the $i$ th to the $j$ th island. $E_{J}=\hbar I_{c} /(2 e)$ is the Josephson coupling energy, and the second sum runs over distinct nearest-neighbor pairs of islands $i$ and $j . n_{i}$ and $\phi_{i}$ are canonically conjugate variables with commutation relations

$$
\left[n_{i}, \phi_{i}\right]=-i \text {. }
$$

For simplicity, we assume no disorder so that $U$ and $E_{J}$ are independent of island or junction coordinates. In the discussion below, we may assume that the array is very large so that we never need to consider its boundaries.

The Hamiltonian (1) can be written in an alternate form involving the time derivatives $\dot{\phi}_{i}$ rather than the number operators $n_{i}$. To do this, we use Eq. (2) and the Heisenberg equation of motion

$$
\dot{\phi}_{i}=\frac{i}{\hbar}\left[H, \phi_{i}\right]=\frac{U}{\hbar} n_{i}
$$

to rewrite $H$ as

$$
H=\sum_{i=1}^{N} \frac{\hbar^{2}}{2 U} \dot{\phi}_{i}^{2}+E_{J} \sum_{\langle i j\rangle}\left[1-\cos \left(\phi_{i}-\phi_{j}\right)-\frac{I_{i j}}{I_{c}}\left(\phi_{i}-\phi_{j}\right)\right] .
$$

Hamiltonian (4) resembles that of an array of coupled anharmonic oscillators. In the limit of small amplitude and zero applied current, the long-wavelength, low-frequency modes of Eq. (4) have the dispersion relation $\hbar \omega_{k}$ $\sim \sqrt{U E_{J}} k b$, where $b$ is the lattice constant and $k$ the wave number. These excitations have previously been called "phase phonons." 45 In the presence of a finite $I$, the lowfrequency, long-wavelength excitations of this Hamiltonian are expected to have a similar dispersion relation.

\section{B. Variational calculation for a diagonal current on a square lattice}

Hamiltonian (1) is characterized by the parameters $E_{J}, U$, and the quantities $I_{i j} / I_{c}$. Depending on the values of these parameters, we expect that the ground state will be either $S$ or insulating (I). We will find the S-I phase boundary approximately by developing a variational approximation for the ground state of this Hamiltonian. The method we choose is a generalization of an earlier approach ${ }^{45}$ used for the case $I=0$. In this section, we find the phase diagram for an applied diagonal current on a square lattice $(\theta=\pi / 4)$.

The starting point for our approximate method is the Gibbs-Bogoliubov inequality. ${ }^{46}$ This inequality states that the Helmholtz free energy $F$ of the system satisfies 


$$
F \leq F_{\text {var }} \equiv F_{0}+\left\langle\left(H-H_{0}\right)\right\rangle_{0},
$$

where $F_{0}$ is the Helmholtz free energy corresponding to some trial Hamiltonian $H_{0}$ and $\langle\ldots\rangle_{0}$ represents an average over the ensemble defined by the Hamiltonian $H_{0}$. At temperature $T=0, F$ and $F_{v a r}$ are the exact and variational energies. For diagonal current injection, we assume that the current applied along either the $x$ or the $y$ direction between adjacent islands is $I_{i j}=I / \sqrt{2}$ (see Fig. 1). The exact Hamiltonian thus takes the form

$$
H=\sum_{i} \frac{\hbar^{2}}{2 U} \dot{\phi}_{i}^{2}+E_{J} \sum_{\langle i j\rangle}\left[1-\cos \left(\phi_{i}-\phi_{j}\right)-\frac{1}{\sqrt{2}} \frac{I}{I_{c}}\left(\phi_{i}-\phi_{j}\right)\right],
$$

where the sum runs over distinct pairs of nearest-neighbor islands and in the last term the island $j$ is either above or to the right of island $i$.

To carry out the variational calculation, it is convenient to choose $H_{0}$ to be a harmonic Hamiltonian of the form

$$
H_{0}=\sum_{i=1}^{N} \frac{\hbar^{2}}{2 U} \dot{\phi}_{i}^{2}+\frac{E_{t}^{2}}{2 E_{J}} \sum_{\langle i j\rangle}\left(\phi_{i}-\phi_{j}-\frac{1}{\sqrt{2}} \frac{\hbar I}{2 e E_{t}}\right)^{2},
$$

where $E_{t}$ represents a trial energy which will be used as the variational parameter. The variational method consists of (i) minimizing the right-hand side of Eq. (5) with respect to the trial parameter $E_{t}$ and then (ii) using the resulting minimum upper bound, and corresponding variational parameters, as an optimized description of the ground state of $H$. We will first carry out this calculation at temperature $T=0$ and later extend the calculation to finite $T$.

Before proceeding, we note one important difference between the calculation at $I=0$ and that at finite $I$. While the eigenvalue spectrum of the Hamiltonian $H$ is bounded below for $I=0$, the corresponding spectrum at finite $I$ is not. The reason is that the term $-E_{J} \Sigma_{\langle i j\rangle}\left(I_{i j} / I_{c}\right)\left(\phi_{i}-\phi_{j}\right)$ can be arbitrarily large and negative. A corresponding term exists even in the Hamiltonian of a single junction with the result that there are no true bound states of that Hamiltonian; instead, there are metastable states from which the phase "particle" can escape by tunneling through a potential barrier. The same is true for the present Hamiltonian (1) although the effective potential is now many dimensional. The effect of this unboundedness is that the resulting variational state will also be metastable - that is, it will be stable with respect to small changes in the variational parameter but unstable with respect to large ones. We will interpret the presence of a metastable minimum as indicating that a superconducting (zerovoltage) state is preferred while the absence of such a minimum will be viewed as indicating that the finite-voltage state is the only one possible. By finding where the superconducting state is metastable, we will obtain a kind of phase boundary between superconducting and nonsuperconducting states at finite current.

We now turn to the details of the variational calculation. It is convenient to introduce transformed variables

$$
\widetilde{\phi}_{i}=\phi_{i}+\lambda_{i},
$$

where $\lambda_{i}$ can be defined so as to simplify the difference in potential terms in Eq. (7). Specifically, we choose $\lambda_{i}-\lambda_{j}$ $=C$, where $C$ is a constant to be determined. We temporarily label the sites on the square lattice by integers $(m, n)$ denoting the $x$ and $y$ coordinates of a lattice site, and associate each site label $i$ with a pair of coordinates $(m, n)$. Then choosing

$$
\lambda_{m, n}=(m+n) C
$$

gives_us $\lambda_{m+1, n}-\lambda_{m, n}=\lambda_{m, n+1}-\lambda_{m, n}=C$. If we choose $C$ $=1 / \sqrt{2} \hbar I /\left(2 e E_{t}\right)$, we will have

$$
\widetilde{\phi}_{i}-\widetilde{\phi}_{j}=\phi_{i}-\phi_{j}-\frac{1}{\sqrt{2}} \frac{\hbar I}{2 e E_{t}} .
$$

The trial Hamiltonian $H_{0}$ is now quadratic in the transformed variables with no linear terms and may be written

$$
H_{0}=\sum_{i=1}^{N} \frac{\hbar^{2}}{2 U} \dot{\widetilde{\phi}}_{i}^{2}+\frac{E_{t}^{2}}{2 E_{J}\langle i j\rangle}\left(\widetilde{\phi}_{i}-\widetilde{\phi}_{j}\right)^{2} .
$$

The average $\left\langle H-H_{0}\right\rangle_{0}$ takes the form

$$
\begin{aligned}
\left\langle H-H_{0}\right\rangle_{0}= & E_{J}\left\langle\sum _ { \langle i j \rangle } \left[ 1-\cos \left(\widetilde{\phi}_{i}-\widetilde{\phi}_{j}+\frac{\hbar \sqrt{2} I}{4 e E_{t}}\right)\right.\right. \\
& \left.\left.-\frac{I}{\sqrt{2} I_{c}}\left(\widetilde{\phi}_{i}-\widetilde{\phi}_{j}+\frac{\hbar \sqrt{2} I}{4 e E_{t}}\right)\right]\right\rangle_{0} \\
& -\frac{E_{t}^{2}}{2 E_{J}}\left\langle\sum_{\langle i j\rangle}\left(\widetilde{\phi}_{i}-\widetilde{\phi}_{j}\right)^{2}\right\rangle_{0} .
\end{aligned}
$$

The variational Hamiltonian $H_{0}$ [Eq. (11)] can now be seen to be that of an ensemble of coupled harmonic oscillators. The two terms represents the kinetic and the potential energies. The phase velocity $\dot{\tilde{\phi}}_{i}$ can be connected to the corresponding number operator $n_{i}$ by Eq. (3).

To diagonalize $H_{0}$, we introduce operators

$$
p_{i}=\hbar n_{i}=m \dot{\phi}_{i}
$$

whose commutator with the operator $\phi_{i}$ is

$$
\left[p_{i}, \phi_{i}\right]=-i \hbar .
$$

From Eqs. (3), (13), and (14), we see that

$$
m=\frac{\hbar^{2}}{U} .
$$

To diagonalize $H_{0}$, we introduce the Fourier transform

$$
\widetilde{\phi}_{i}=\frac{1}{\sqrt{N}} \sum_{\mathbf{k}} \exp \left(-i \mathbf{k} \cdot \mathbf{R}_{i}\right) \widetilde{\phi}_{\mathbf{k}},
$$

where $\mathbf{R}_{i}$ is the position vector of the $i$ th superconducting island and the sum runs over the first Brillouin zone of the grain lattice. We also introduce the transform 


$$
p_{i}=\frac{1}{\sqrt{N}} \sum_{\mathbf{k}} \exp \left(i \mathbf{k} \cdot \mathbf{R}_{i}\right) \Pi_{\mathbf{k}}
$$

In terms of these variables, it can be shown that $H_{0}$ can be written as

$$
H_{0}=\sum_{k}\left(\frac{\Pi_{k}^{\dagger} \Pi_{k}}{2 m}+\frac{1}{2} m \omega_{k}^{2} \widetilde{\phi}_{\mathbf{k}}^{\dagger} \widetilde{\phi}_{\mathbf{k}}\right)
$$

where the harmonic frequencies $\omega_{\mathbf{k}}$ are given by

$$
\omega_{\mathbf{k}}=\sqrt{2} \omega \sqrt{\sum_{\delta} \sin ^{2}(\mathbf{k} \cdot \delta / 2)},
$$

where now the sum runs over all (both positive and negative) nearest-neighbor vectors $\delta$ to a given lattice site. The quantity $\omega^{2}$ is given by

$$
\omega^{2}=\frac{U E_{t}^{2}}{\hbar^{2} E_{J}}
$$

The derivation of these results is very similar to that given in Ref. 45 for the case of zero applied current.

Next, we turn to $\left\langle H-H_{0}\right\rangle_{0}$. Since $H_{0}$ is harmonic in the transformed variables $\widetilde{\phi}_{i}$ with no linear terms, the linear terms in the $\widetilde{\phi}_{i}$ 's in Eq. (12) will not contribute to $\left\langle H-H_{0}\right\rangle_{0}$. Furthermore, because $H_{0}$ is harmonic, the expectation value of the cosine term can be simplified by using a well-known identity (see, for example, Ref. 47), which applies whenever the variational Hamiltonian is quadratic in the $\widetilde{\phi}_{i}$, namely,

$$
\left\langle\cos \left(\widetilde{\phi}_{i}-\widetilde{\phi}_{j}\right)\right\rangle_{0}=\exp \left[-\frac{1}{2}\left\langle\left(\widetilde{\phi}_{i}-\widetilde{\phi}_{j}\right)^{2}\right\rangle_{0}\right] \text {. }
$$

Using eq. (21), we obtain

$$
\begin{aligned}
\left\langle H-H_{0}\right\rangle_{0}= & E_{J} \sum_{\langle i j\rangle}\left\{1-\cos \left(\frac{\hbar \sqrt{2} I}{4 e E_{t}}\right) \exp \left[-\frac{1}{2}\left\langle\left(\widetilde{\phi}_{i}-\widetilde{\phi}_{j}\right)^{2}\right\rangle_{0}\right]\right. \\
& \left.-\frac{I}{I_{c}} \frac{\hbar I}{4 e E_{t}}\right\}-\frac{E_{t}^{2}}{2 E_{J}} \sum_{\langle i j\rangle}\left\langle\left(\widetilde{\phi}_{i}-\widetilde{\phi}_{j}\right)^{2}\right\rangle_{0}
\end{aligned}
$$

To evaluate the quantity $D_{i j} \equiv\left\langle\left(\widetilde{\phi}_{i}-\widetilde{\phi}_{j}\right)^{2}\right\rangle_{0}$ in expression (22), we use the Fourier transform in Eq. (16) to obtain

$$
D_{i j}=\frac{1}{N}\left\langle\left|\sum_{k} \widetilde{\phi}_{k}\left(e^{i \vec{k} \cdot \vec{R}_{i}}-e^{i \vec{k} \cdot \vec{R}_{j}}\right)\right|^{2}\right\rangle_{0},
$$

where the sum again runs over all $\mathbf{k}$ vectors in the first Brillouin zone.

Then, since the Hamiltonian $H_{0}$, from Eq. (18), is that of a collection of independent harmonic oscillators, Eq. (23) simplifies to

$$
\begin{aligned}
D_{i j} & =4 \sum_{k}\left\langle\tilde{\phi}_{\mathbf{k}} \tilde{\phi}_{-\mathbf{k}}\right\rangle_{0} \sin ^{2}\left(\mathbf{k} \cdot \mathbf{R}_{i j} / 2\right) \\
& =4 \sum_{\mathbf{k}} \sin ^{2}\left(\mathbf{k} \cdot \mathbf{R}_{i j} / 2\right) \frac{\hbar}{2 m \omega_{k}}\left(2 n_{k}+1\right) .
\end{aligned}
$$

In Eq. (24), $\omega_{k}$ and $m$ are the frequency and the effective mass of the "phase phonons," as calculated above, and $n_{k}$ is the corresponding Bose occupation number, given at temperature $T$ by $n_{k}=1 /\left[\exp \left(\hbar \omega_{k} / k_{B} T\right)-1\right]$. At $T=0$, we have $n_{k}=0$.

Inserting Eq. (19) into Eq. (24), and converting the sum over $\mathbf{k}$ into an integral, we arrive at the $T=0$ expression

$$
\left\langle\left(\tilde{\phi}_{i}-\widetilde{\phi}_{j}\right)^{2}\right\rangle_{0}=\frac{\hbar}{m} \frac{v_{c}}{2 \pi^{2}} \int_{B Z} d^{2} k \frac{\sin ^{2}\left(\mathbf{k} \cdot \mathbf{R}_{i j} / 2\right)}{\omega_{\mathbf{k}}},
$$

where $v_{c}$ is the area of one unit cell of the grain lattice and the integral runs over the first Brillouin zone.

Equation (25) is, in fact, applicable to a general lattice in two dimensions. Specializing to a square lattice with lattice constant $b$, and considering $\mathbf{R}_{i j}=b \hat{\mathbf{x}}$, we may rewrite Eq. (19) as

$$
\omega_{\mathbf{k}}=2 \omega \sqrt{\sin ^{2}\left(k_{x} b / 2\right)+\sin ^{2}\left(k_{y} b / 2\right)} .
$$

Similarly, eq. (25) becomes

$$
\begin{aligned}
& \left\langle\left(\widetilde{\phi}_{i}-\widetilde{\phi}_{j}\right)^{2}\right\rangle_{0} \\
& =\frac{\hbar}{m \omega}\left(\frac{b}{2 \pi}\right)^{2} \int_{-\pi / b}^{\pi / b} \int_{-\pi / b}^{\pi / b} \frac{\sin ^{2}\left(\frac{k_{x} b}{2}\right) d k_{x} d k_{y}}{\sqrt{\sin ^{2}\left(\frac{k_{x} b}{2}\right)+\sin ^{2}\left(\frac{k_{y} b}{2}\right)}} .
\end{aligned}
$$

The prefactor in this last equation can be simplified using Eqs. (15) and (20), and the integral evaluated numerically. The final result is

$$
\left\langle\left(\widetilde{\phi}_{i}-\widetilde{\phi}_{j}\right)^{2}\right\rangle_{0}=\alpha \sqrt{\frac{U E_{J}}{E_{t}^{2}}},
$$

where $\alpha=0.479$.

Combining all the terms, we can now obtain an explicit expression for the desired quantity $\left\langle H-H_{0}\right\rangle_{0}$ for a square lattice with current injected along the diagonal. The result is

$$
\begin{aligned}
\left\langle H-H_{0}\right\rangle_{0}= & 2 N E_{J}\left[\left(1-\cos \left(\frac{\sqrt{2} I E_{J}}{2 I_{c} E_{t}}\right) e^{-\alpha / 2 \sqrt{U E_{J} / E_{t}^{2}}}\right.\right. \\
& \left.-\frac{1}{2}\left(\frac{I}{I_{c}}\right)^{2} \frac{E_{J}}{E_{i}}\right]-E_{J} N \alpha \sqrt{\frac{U E_{t}^{2}}{E_{J}^{3}}} .
\end{aligned}
$$

Finally, we need the free energy of the reference Hamiltonian $H_{0}$. This may be obtained straightforwardly since $H_{0}$ is harmonic. At $T=0$, it is just the sum of the zero-point energies $\hbar \omega_{k} / 2$ for the reference harmonic oscillators. Once again converting the sum over $\mathbf{k}$ to an integral, we find that

$$
F_{0} \equiv E_{0}=\sqrt{\frac{E_{t}^{2} U}{E_{J}}}\left(\frac{b}{2 \pi}\right)^{2} \int_{-\pi / b}^{\pi / b} \int_{-\pi / b}^{\pi / b} \sqrt{\sin ^{2}\left(\frac{k_{x} b}{2}\right)+\sin ^{2}\left(\frac{k_{y} b}{2}\right)} d k_{x} d k_{y}
$$


where we use the fact that the free energy $F_{0}$ is just equivalent to the energy $E_{0}$ at $T=0$ The integral on the right-hand side can be shown to be equal to twice the integral in Eq. (27), and one finds that, for the present case of $2 N$ junctions, this zero-point energy is

$$
E_{0}=(2 N) 2 \alpha \sqrt{\frac{E_{t}^{2} U}{E_{J}}} .
$$

Combining all terms, we now have a complete expression for the variational free energy at $T=0$ written entirely in terms of the variational parameter $E_{t}$. Scaling the free energy by the number of junctions $2 N$ and the parameter $E_{J}$, and introducing suitable dimensionless variables, we obtain

$$
\frac{F_{v a r}}{2 N E_{J}}=\left(\frac{3 \alpha}{2}\right) \sqrt{a x^{2}}+1-\cos \left(\sqrt{2} \frac{I_{r}}{2 x}\right) \exp \left(-\frac{1}{2} \alpha \sqrt{\frac{a}{x^{2}}}\right)-\frac{I_{r}^{2}}{2 x},
$$

where $x=E_{t} / E_{J}, a=U / E_{J}$, and $I_{r}=I / I_{c}$.

\section{Generalization to nondiagonal bias currents}

The results of the previous section are readily generalized to a bias current which forms an arbitrary angle $\theta$ with the $x$ axis. The Hamiltonian still has the form

$H=\frac{U}{2} \sum_{i 1}^{N}\left(\frac{\hbar}{2 U}\right)^{2} \dot{\phi}_{i}^{2}+E_{J} \sum_{\langle i j\rangle}\left[1-\cos \left(\phi_{i}-\phi_{j}\right)-\frac{I_{i j}}{I_{c}}\left(\phi_{i}-\phi_{j}\right)\right]$,

where $I_{i j}$ still represents the bias current between the $i$ th and $j$ th superconducting island. For the case now under consideration, we write $I_{i j}=I \cos \theta$ or $I \sin \theta$ for junctions parallel to the $x$ and $y$ axes.

To carry out the variational calculation, we choose the trial Hamiltonian, and make the corresponding change of variables to $\widetilde{\phi}_{i}$, as before, but with $I_{i j}$ different for the $x$ and $y$ directions. Once again, the terms linear in the $\widetilde{\phi}_{i}$ 's do not contribute to the expectation values taken with respect to the reference Hamiltonian, and the other simplifications leading to Eq. (29) apply here also. The expression for $\left\langle H-H_{0}\right\rangle_{0}$ is now found to be

$$
\begin{aligned}
& E_{J} \sum_{\langle i j\rangle}\left[1-\cos \left(\frac{\hbar I_{i j}}{2 e E_{t}}\right) \exp \left(-\frac{1}{2}\left\langle\left(\widetilde{\phi}_{i}-\widetilde{\phi}_{j}\right)^{2}\right\rangle_{0}\right)-\frac{I_{i j}}{I_{c}}\left(\frac{\hbar I_{i j}}{2 e E_{t}}\right)\right] \\
& -\frac{E_{t}^{2}}{2 E_{J}} \sum_{\langle i j\rangle}\left\langle\left(\widetilde{\phi}_{i}-\widetilde{\phi}_{j}\right)^{2}\right\rangle_{0} .
\end{aligned}
$$

The final expression for the variational free energy (i.e., at $T=0$ the energy) is found to be

$$
\begin{aligned}
\frac{F_{v a r}}{2 N E_{J}}= & \frac{3 \alpha}{2} \sqrt{a x^{2}}+1-\frac{1}{2}\left[\cos \left(\frac{I_{r} \cos \theta}{x}\right)\right. \\
& \left.+\cos \left(\frac{I_{r} \sin \theta}{x}\right)\right] \exp \left(-\frac{1}{2} \alpha \sqrt{\frac{a}{x^{2}}}\right)-\frac{I_{r}^{2}}{2 x} .
\end{aligned}
$$

The dimensionless variables $x, a$, and $I_{r}$ are the same as in the case of the diagonal bias current. Setting $\theta=\pi / 4$ in Eq. (35) reduces this expression to our previous result in Eq. (32) for a diagonal current.

\section{Extension to a simple cubic lattice}

These results can be easily generalized to a simple [threedimensional (3D)] cubic lattice. We assume that the bias current runs along the body diagonal of the cube and define $I$ such that the bias current along a bond along the $x, y$, or $z$ axes is $I / \sqrt{3}$. We define $I_{r}=I / I_{c}$ as before. The Hamiltonian is still given by Eq. (1). The total number of lattice points is still denoted $N$. The dispersion relation in Eq. (19) becomes

$$
\omega_{k}=2 \omega \sqrt{\sin ^{2}\left(k_{x} b / 2\right)+\sin ^{2}\left(k_{y} b / 2\right)+\sin ^{2}\left(k_{z} b / 2\right)} .
$$

The quantity $\left\langle\left(\widetilde{\phi}_{i}-\widetilde{\phi}_{j}\right)^{2}\right\rangle_{0}$ is now given by

$$
\left\langle\left(\widetilde{\phi}_{i}-\widetilde{\phi}_{j}\right)^{2}\right\rangle_{h}=\sqrt{\frac{U E_{J}}{E_{t}^{2}}}\left(\frac{b}{2 \pi}\right)^{3} \int_{-\pi / b}^{\pi / b} \int_{-\pi / b}^{\pi / b} \int_{-\pi / b}^{\pi / b} \frac{\sin ^{2}\left(\frac{k_{x} b}{2}\right) d k_{x} d k_{y} d k_{z}}{\sqrt{\sin ^{2}\left(\frac{k_{x} b}{2}\right)+\sin ^{2}\left(\frac{k_{y} b}{2}\right)+\sin ^{2}\left(\frac{k_{z} b}{2}\right)}}
$$

and can be evaluated numerically with the result

$$
\left\langle\left(\widetilde{\phi}_{i}-\widetilde{\phi}_{j}\right)^{2}\right\rangle_{0}=\beta \sqrt{\frac{U E_{J}}{E_{t}^{2}}},
$$

where $\beta=0.398$.

The steps to obtain the free energy (i.e., at $T=0$ the energy) are identical to those in two-dimensional (2D). The free energy of the reference Hamiltonian $H_{0}$ becomes

$$
E_{0}=\sqrt{\frac{U E_{t}^{2}}{E_{J}}}\left(\frac{b}{2 \pi}\right)^{3} \int_{-\pi / b}^{\pi / b} \int_{-\pi / b}^{\pi / b} \int_{-\pi / b}^{\pi / b} \sqrt{\sin ^{2}\left(\frac{k_{x} b}{2}\right)+\sin ^{2}\left(\frac{k_{y} b}{2}\right)+\sin ^{2}\left(\frac{k_{z} b}{2}\right)} d k_{x} d k_{y} d k_{z} .
$$




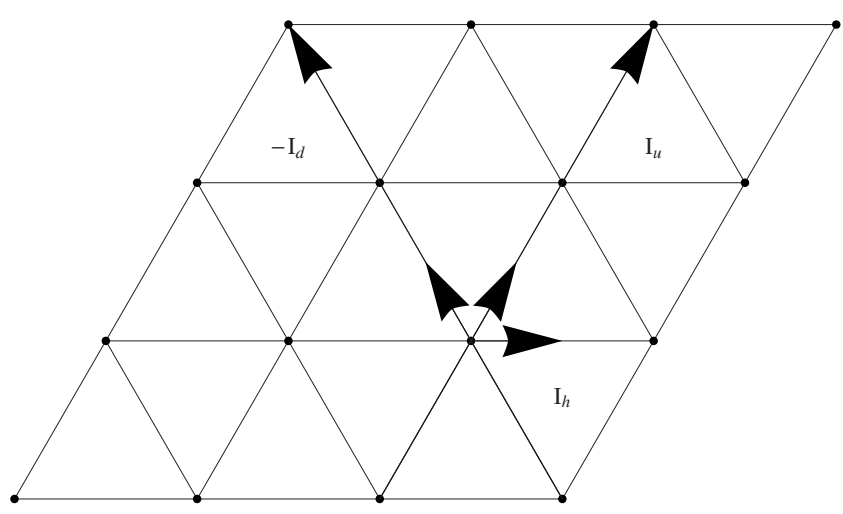

FIG. 2. Schematic of the geometry for a triangular lattice. Currents $I_{u}, I_{d}$, and $I_{h}$ are applied to junctions along the $u, d$, and $h$ directions, as indicated. For a net current in the horizontal direction, we choose $I_{h}=I$ and $I_{u}=I_{d}=0$. For a net current in the vertical direction, we choose $I_{u}=-I_{d}=I \sqrt{3} / 2$ with $I_{h}=0$.

This integral can be shown to equal three times that in Eq. (37)

$$
E_{0}=3 \beta \sqrt{\frac{U E_{t}^{2}}{E_{J}}} .
$$

Again defining $a=U / E_{J}, x=E_{t} / E_{J}$, and $I_{r}=I / I_{c}$, we obtain the final result

$$
\frac{F_{v a r}}{3 N E_{J}}=\left(\frac{5}{2} \beta\right) \sqrt{a x^{2}}+1-\cos \left(\frac{I_{r}}{\sqrt{3} x}\right) \exp \left(-\frac{1}{2} \beta \sqrt{\frac{a}{x^{2}}}\right)-\frac{I_{r}^{2}}{3 x} .
$$

\section{E. Extension to a triangular lattice}

Next, we consider a triangular lattice of superconducting islands whose nearest neighbors are coupled by identical Josephson junctions of critical current $I_{c}$. The system is still described by the Hamiltonian (1) and (4). The geometry is now shown in Fig. 2. The number of islands is still denoted by $N$, but there are now three distinct bond directions in the lattice, corresponding to three applied bond currents. We label these $I_{u}, I_{d}$, and $I_{h}$ (note that $-I_{d}$ is indicated in Fig. 2). The applied current density, however, has only two components, say $J_{x}$ and $J_{y}$, in the $x$ and $y$ directions. Thus, specifying $J_{x}$ and $J_{y}$ is not sufficient to determine $I_{u}, I_{d}$, and $I_{h}$ uniquely. In our numerical results given below, we describe two reasonable choices for these currents. One might express these choices in terms of current densities but it seems more transparent to specify the current across each nearestneighbor bond.

We again wish to obtain a variational approximation to the ground state of this system, using the Gibbs-Bogoliubov inequality. As before, we use a trial Hamiltonian of the form

$$
H_{0}=\sum_{i=1}^{N} \frac{\hbar^{2}}{2 U} \dot{\phi}_{i}^{2}+\frac{E_{t}^{2}}{2 E_{J}} \sum_{\langle i j\rangle}\left(\phi_{i}-\phi_{j}-\frac{\hbar I_{i j}}{2 e E_{t}}\right)^{2},
$$

where $E_{t}$ is a variational parameter. We again introduce the transformed variables

$$
\widetilde{\phi}_{i}=\phi_{i}+\lambda_{i},
$$

where $\lambda_{i}$ can be defined to simplify the difference in potential terms in Eq. (42). It can be show that there exists a choice of $\lambda_{i}$ such that

$$
\widetilde{\phi}_{i}-\widetilde{\phi}_{j}=\phi_{i}-\phi_{j}-\frac{\hbar I_{i j}}{2 e E_{t}}
$$

Because this transformation is possible, $H_{0}$ does take the form of Eq. (11), as we require. In terms of the transformed variables, again using the identity (21), the expectation value $\left\langle H-H_{0}\right\rangle_{0}$ can still be written as

$$
\begin{aligned}
\left\langle H-H_{0}\right\rangle_{0}= & E_{J} \sum_{\langle i j\rangle}\left[1-\cos \left(\frac{\hbar I_{i j}}{2 e E_{t}}\right) \exp \left(-\frac{1}{2}\left\langle\left(\widetilde{\phi}_{i}-\widetilde{\phi}_{j}\right)^{2}\right\rangle_{0}\right)\right. \\
& \left.-\left(\frac{I_{i j}}{I_{c}}\right) \frac{\hbar I_{i j}}{2 e E_{t}}\right]-\frac{E_{t}^{2}}{2 E_{J}} \sum\left\langle\left(\widetilde{\phi}_{i}-\widetilde{\phi}_{j}\right)^{2}\right\rangle_{0}
\end{aligned}
$$

The expectation value of the square of the phase difference can be expressed in terms of suitable Fourier-transformed variables

$$
\left\langle\left(\widetilde{\phi}_{i}-\widetilde{\phi}_{j}\right)^{2}\right\rangle_{0}=4 \sum_{\mathbf{k}}\left\langle\widetilde{\phi}_{\mathbf{k}} \widetilde{\phi}_{-\mathbf{k}}\right\rangle_{0} \sin ^{2}\left(\frac{\mathbf{k} \cdot \mathbf{R}_{i j}}{2}\right),
$$

where the sum runs over the Brillouin zone of the grain lattice and the Fourier transform $\widetilde{\phi}_{\mathbf{k}}$ is related to $\widetilde{\phi}_{i}$ by Eq. (16). For any value of the nearest-neighbor vector $\mathbf{R}_{i j}$, this sum becomes

$$
\left\langle\left(\widetilde{\phi}_{i}-\widetilde{\phi}_{j}\right)^{2}\right\rangle_{0}=4 \sum_{\mathbf{k}} \sin ^{2}\left(\frac{\mathbf{k} \cdot \mathbf{R}_{i j}}{2}\right) \frac{\hbar}{2 m \omega_{k}}\left(2 n_{k}+1\right),
$$

where $m$ the effective mass of the phase phonons and $n_{k}$ is the Bose occupation number, as before.

We restrict ourselves to $T=0$, so that $n_{k}=0$. For a triangular lattice, the dispersion relation is

$$
\omega_{k}=2 \omega \sqrt{\sin ^{2}\left(\frac{k_{x} b}{2}\right)+\sin ^{2}\left(\frac{k_{x} b}{4}+\frac{\sqrt{3} k_{y} b}{4}\right)+\sin ^{2}\left(\frac{k_{x} b}{4}-\frac{\sqrt{3} k_{y} b}{4}\right)} .
$$

Inserting Eq. (48) into Eq. (40), and converting the sum over $\mathbf{k}$ into an integral, we obtain 


$$
\left\langle\left(\widetilde{\phi}_{i}-\widetilde{\phi}_{j}\right)^{2}\right\rangle_{0}=\frac{\hbar}{m \omega A} \iint^{B Z} \frac{\sin ^{2}\left(\frac{\vec{k} \cdot \overrightarrow{R_{i j}}}{2}\right) d k_{x} d k_{y}}{\sin ^{2}\left(\frac{k_{x} b}{2}\right)+\sin ^{2}\left(\frac{k_{x} b}{4}+\frac{\sqrt{3} k_{y} b}{4}\right)+\sin ^{2}\left(\frac{k_{x} b}{4}-\frac{\sqrt{3} k_{y} b}{4}\right)},
$$

where the integral is over the first Brillouin zone, $A$ is the zone area, and $\mathbf{R}_{i j}$ is one of the nearest-neighbor vectors. The integral has the same value for any choice of $\mathbf{R}_{i j}$. The prefactor can be simplified using Eqs. (15) and (20), and the integral can be calculated numerically, with the result

$$
\left\langle\left(\widetilde{\phi}_{i}-\widetilde{\phi}_{j}\right)^{2}\right\rangle_{0}=\gamma \sqrt{\frac{U E_{J}}{E_{t}^{2}}},
$$

where $\gamma=0.395$.

Combining all the terms, we can now obtain an explicit expression for the desired quantity $\left\langle H-H_{0}\right\rangle_{0}$. The result is

$$
\left\langle H-H_{0}\right\rangle_{0}=\left(N E_{J}\right)\left\{3-e^{-\gamma / 2 \sqrt{U E_{J} / E_{t}^{2}}}\left[\cos \left(\frac{I_{h} E_{J}}{I_{c} E_{t}}\right)+\cos \left(\frac{I_{u} E_{J}}{I_{c} E_{t}}\right)+\cos \left(\frac{I_{d} E_{J}}{I_{c} E_{t}}\right)\right]\right\}-\left(N E_{J}\right)\left(\frac{I_{h}^{2}}{I_{c}^{2}}+\frac{I_{u}^{2}}{I_{c}^{2}}+\frac{I_{d}^{2}}{I_{c}^{2}}\right) \frac{E_{J}}{E_{t}}-\left(3 E_{J} N \gamma / 2\right) \sqrt{\frac{U E_{t}^{2}}{E_{J}^{3}}},
$$

where $I_{h}, I_{u}$, and $I_{d}$ represent the currents in the three distinct nearest-neighbor bonds as discussed earlier.

Finally, we need the free energy (equivalent at $T=0$ to the zero-point energy) of the reference Hamiltonian $H_{0}$. The calculation is straightforward and analogous to those of the previous sections with the result

$$
F_{0} \equiv E_{0}=\sqrt{\frac{E_{t}^{2} U}{E_{J}}}\left(\frac{1}{A}\right) \times \iint^{B Z} d k_{x} d k_{y} \sqrt{\sin ^{2}\left(\frac{k_{x} b}{2}\right)+\sin ^{2}\left(\frac{k_{x} b}{4}+\frac{\sqrt{3} k_{y} b}{4}\right)+\sin ^{2}\left(\frac{k_{x} b}{4}-\frac{\sqrt{3} k_{y} b}{4}\right)} .
$$

The integral can be shown to equal exactly three times that of Eq. (49). Thus, for $3 N$ junctions, this zero-point energy is

$$
E_{0}=9 N \gamma \sqrt{\frac{E_{t}^{2} U}{E_{J}}} .
$$

Combining all terms, we now have a complete expression for the variational free energy written entirely in terms of the variational energy $E_{t}$. Scaling the free energy by the number of junctions $3 N$ and the parameter $E_{J}$, and introducing suitable dimensionless variables, we obtain

$$
\begin{aligned}
\frac{F_{v a r}}{3 N E_{J}}= & \frac{5 \gamma}{2} \sqrt{a x^{2}}+1-e^{-\gamma / 2 \sqrt{a / x^{2}}} \times \frac{1}{3}\left[\cos \left(\frac{I_{h}}{x I_{c}}\right)+\cos \left(\frac{I_{u}}{x I_{c}}\right)\right. \\
& \left.+\cos \left(\frac{I_{d}}{x I_{c}}\right)\right]-\frac{I_{h}^{2}+I_{u}^{2}+I_{d}^{2}}{3 x I_{c}^{2}}
\end{aligned}
$$

where $x=E_{t} / E_{J}$ and $a=U / E_{J}$.

\section{F. Temperature dependence}

Thus far all calculations have been carried out at temperature $T=0$. At finite $T$, the principal differences in the calculation occur in the occupation number $n_{k}$, and the free energy of the reference Hamiltonian. The analog of Eq. (24) at finite $T$ gives the expectation value of the square of the phase difference, $\left\langle\left(\widetilde{\phi}_{i}-\widetilde{\phi}_{j}\right)^{2}\right\rangle_{T}$, which we call $D_{i j}(T)$

$$
D_{i j}(T)=4 \sum_{\mathbf{k}} \sin ^{2}\left(k_{x} b / 2\right) \frac{\hbar}{2 m \omega_{k}} \operatorname{coth}\left(\beta \hbar \omega_{k} / 2\right),
$$

where we use $n_{k}=1 /\left(e^{\beta \hbar \omega}-1\right)$ and $\beta=1 /\left(k_{B} T\right)$. We limit ourselves to the case of a square lattice. This sum can be converted to an integral as at $T=0$ with the result

$$
\begin{aligned}
D_{i j}(T)= & {[b /(2 \pi)]^{2}\left[U E_{J} / E_{t}^{2}\right]^{1 / 2} \times \int_{-\pi / b}^{\pi / b} \int_{-\pi / b}^{\pi / b} \frac{\sin ^{2}\left(k_{x} b / 2\right)}{\left[\sin ^{2}\left(k_{x} b / 2\right)+\sin ^{2}\left(k_{y} b / 2\right)\right]^{1 / 2}} \operatorname{coth}\left\{( \beta / 2 ) ( U E _ { t } ^ { 2 } / E _ { J } ) ^ { 1 / 2 } \left[\sin ^{2}\left(k_{x} b / 2\right)\right.\right.} \\
& \left.\left.+\sin ^{2}\left(k_{y} b / 2\right)\right]^{1 / 2}\right\} d k_{x} d k_{y} .
\end{aligned}
$$

The corresponding integral for the reference Helmholtz free energy is 


$$
\frac{F_{0}}{2 N E_{J}}=\left(\frac{b}{2 \pi}\right)^{2} \int_{-\pi / b}^{\pi / b} \int_{-\pi / b}^{\pi / b} d k_{x} d k_{y} \times\left\{\sqrt{\frac{U E_{t}^{2}}{E_{J}^{3}}} \sqrt{\sin ^{2}\left(\frac{k_{x} b}{2}\right)+\sin ^{2}\left(\frac{k_{y} b}{2}\right)}+\frac{1}{\beta E_{J}} \log \left[1-e^{\left.-\beta E_{J} \sqrt{U E_{t}^{2} / E_{J}^{3}} \sqrt{\sin ^{2}\left(k_{x} b / 2\right)+\sin ^{2}\left(k_{y} b / 2\right)}\right]}\right\} .\right.
$$

Given $D_{i j}$ and $F_{0}$, the scaled variational free energy $F_{v a r}$ is

$$
\frac{F_{v a r}}{2 N E_{J}}=F_{0}-\frac{1}{2}\left(\frac{E_{t}}{E_{J}}\right)^{2} D_{i j}(T)+1-\cos \left(\frac{\sqrt{2}}{2} \frac{I E_{J}}{E_{t} I_{c}}\right) e^{-1 / 2 D_{i j}(T)}-\frac{1}{2} \frac{I^{2} E_{J}}{I_{c}^{2} E_{t}} .
$$

Just as at $T=0$, this variational free energy has local minima for certain values of the parameters in the Hamiltonian, from which one can derive the superconductor/nonsuperconductor phase diagram as a function of $T$ and those parameters.

\section{NUMERICAL RESULTS AND DISCUSSION}

One obvious characteristic of the free energy in Eq. (32) is that it diverges to negative infinity as $E_{t}$ (or $x=E_{t} / E_{J}$ ) approaches zero. But, as mentioned previously, $F_{\text {var }}$ may also have a local minimum at a nonzero value of $E_{t}$ for some values of $a=U / E_{J}$ and $I_{r}=I / I_{c}$.

In Fig. 3 we plot the free energy $F_{\text {var }}[\mathrm{Eq} .(32)]$ as a function of $E_{t} / E_{J}$ for $I / I_{c}=0.5$ and several values of $U / E_{J}$, assuming current injection along the diagonal. Clearly, the existence of a metastable minimum in this free energy depends on the ratios $U / E_{J}$ and $I / I_{c}$. When there is no such metastable minimum, $F_{\text {var }}$ diverges towards $-\infty$ as $x \rightarrow 0$. We interpret the disappearance of the metastable state as indicating the onset of a finite-voltage state.

Next, we briefly describe the numerical method we used to find the critical values of the ratio $a=U / E_{J}$. For each $I / I_{c}$, a critical $a$ can be found, at which $F_{v a r}\left(a, I_{r}\right)$ ceases to have a local minimum at nonzero $x$. This value was found by first choosing a fixed current $\left(I / I_{c}\right)$ and then scanning through values of the ratio $U / E_{J}$. For each $U / E_{J}$, the program

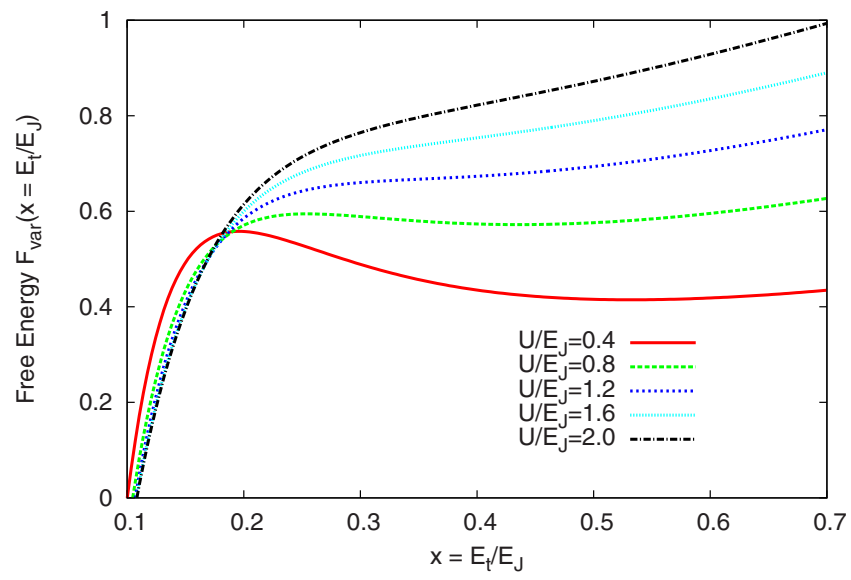

FIG. 3. (Color online) Variational free energy at current $I / I_{c}$ $=0.5$, as a function of the variational parameter $E_{t} / E_{J}$, for a square lattice and various values of $U / E_{J}$ as indicated. The bias current is along the diagonal $\left(\theta=\pi / 4\right.$ with $I_{x}=I_{y}=I / \sqrt{2}$. stepped through different values of $E_{t} / E_{J}$, searching for a sign change in the first derivative of the free energy expression in Eq. (32). The ratio of $U / E_{J}$ was increased until no such sign change was found. Once the largest $U / E_{J}$ was found that still yielded a local free energy minimum, the process was repeated in a narrower range around the first approximation of the critical values. Specifically, the ratio $U / E_{J}$ was initially varied from $10^{-9}$ to 4.5 in 200 equal steps. The ratio $E_{t} / E_{J}$ was also varied from 0.01 to 1.8 , also in 200 equal steps. After the first approximations of the critical values $\left(U / E_{J}\right)_{c}$ and $\left(E_{t} / E_{J}\right)_{c}$ were recorded, the range of each parameter was reduced to $10 \%$ of the original range while the number of steps remained 200. This solution process was repeated through seven iterations to obtain very precise critical values. The entire procedure was carried out for many values of $I / I_{c}$ to obtain the fairly smooth curve shown in Fig. 4. These critical values represent a phase boundary between the superconducting and nonsuperconducting states.

To our knowledge, no theoretical or experimental work has been carried out which can be compared to most of the phase boundary shown in Fig. 4. But the endpoints of the curve can be compared to known values. At high current, in our variational approximation, $\left(U / E_{J}\right)_{c}$ drops to zero at $I / I_{c} \sim 1.4$. That is, when the current $I$ across a plaquette reaches a magnitude of $\sqrt{2} I_{c}$, no superconducting state can exist regardless of the ratio $U / E_{J}$. This factor of $\sqrt{2}$ is clearly

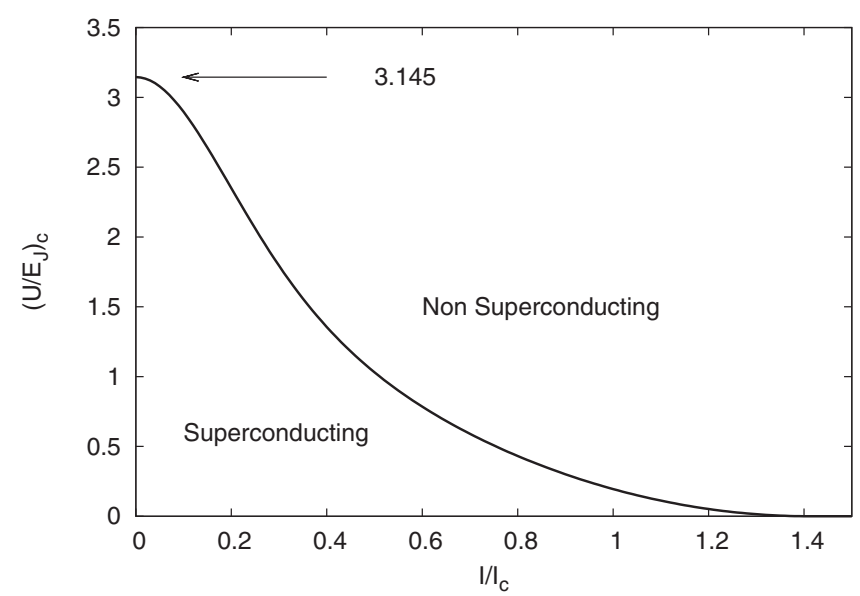

FIG. 4. Critical value $a_{c} \equiv\left(U / E_{J}\right)_{c}$ separating the superconducting and nonsuperconducting phases, as a function of bias current $I / I_{c}$, for the 2D square lattice with current directed along the diagonal as in Fig. 3, and temperature $T=0$. 


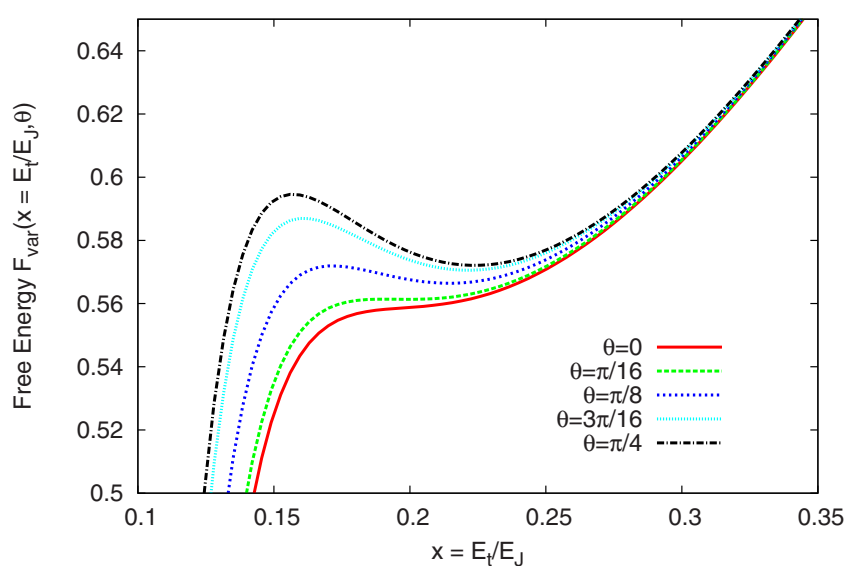

FIG. 5. (Color online) Variational free energy at fixed $U / E_{J}$ $=0.8$ and $I / I_{c}=0.5$, plotted as a function of the scaled variational parameter $E_{t} / E_{J}$, for various angles $\theta$ as indicated. The applied currents per bond in the $x$ and $y$ directions are $I \cos \theta$ and $I \sin \theta$.

what one would expect from applying equal currents along each of the two legs of a right triangle in the square lattice, as in the present case. The other end of the phase boundary represents the zero-current critical value of $U / E_{J}$. This value has been calculated for a square lattice ${ }^{48}$ using $(2+1) D$ quantum Monte Carlo and found to equal $\left(U / E_{J}\right)_{c}=4.84$. This result is extremely close to that given by other Monte Carlo studies of the classical 3D XY model, ${ }^{49}$ to which our 2D quantum model corresponds. This value also appears to agree with the work of Kopeć and José. ${ }^{50}$ Our variational critical value of $\left(U / E_{J}\right)_{c}=3.14$ at $I / I_{c}$ is about a third lower than established value of 4.84. An error of about a third is not surprising in a variational theory of this kind.

The dependence of $F_{v a r}$ on the bias angle is shown in Fig. 5. The variational free energy $F_{v a r}$ is shown as a function of $E_{t} / E_{J}$ for several different bias angles at a fixed $I / I_{c}$ and $U / E_{J}$. These curves were obtained by choosing a specific angle to insert into Eq. (35) along with the fixed ratios. The variational parameter $E_{t} / E_{J}$ was scanned to obtain a nearly continuous curve.

Fig. 5 shows that, for certain values of $I / I_{c}$ (or $U / E_{J}$ ), the metastable superconducting state can be created or destroyed by varying $\theta$. Hence, for fixed $U$ and $E_{J}$, the superconducting to nonsuperconducting transition can be achieved by changing either the magnitude or the direction of the current $I$. For the parameters used in Fig. 5, a current of $I_{c} / 2$ is sufficient to destroy the metastable minimum if all the current is driven through only bonds in the $x$ direction $(\theta=0)$. If the same current is divided between bonds in the $x$ and $y$ directions $(\theta \neq 0)$, the local minimum usually remains, for the parameters used in Fig. 5. This behavior agrees with what is intuitively expected.

In Fig. 6, we plot the critical value of $I / I_{c}$ [which we denote $\left(I / I_{c}\right)_{c}$ on the angle $\theta$ at which the bias current is applied. Clearly, $\left(I / I_{c}\right)_{c}$ depends most strongly on $\theta$ for the smallest $\left.U / E_{J}\right]$. But there is angular dependence for all values of $U / E_{J}$. The curves for $U / E_{J}=1.375$ and 1.75 appear flat in the main figure. But the inset, which shows the scaled difference $\Delta\left(I / I_{c}\right)_{c} \equiv\left[\left(I / I_{c}\right)_{c}(\theta)-\left(I / I_{c}\right)_{c}(\theta=0)\right] /\left(I / I_{c}\right)_{c}(\theta=0)$, makes this $\theta$ dependence obvious even for high charging

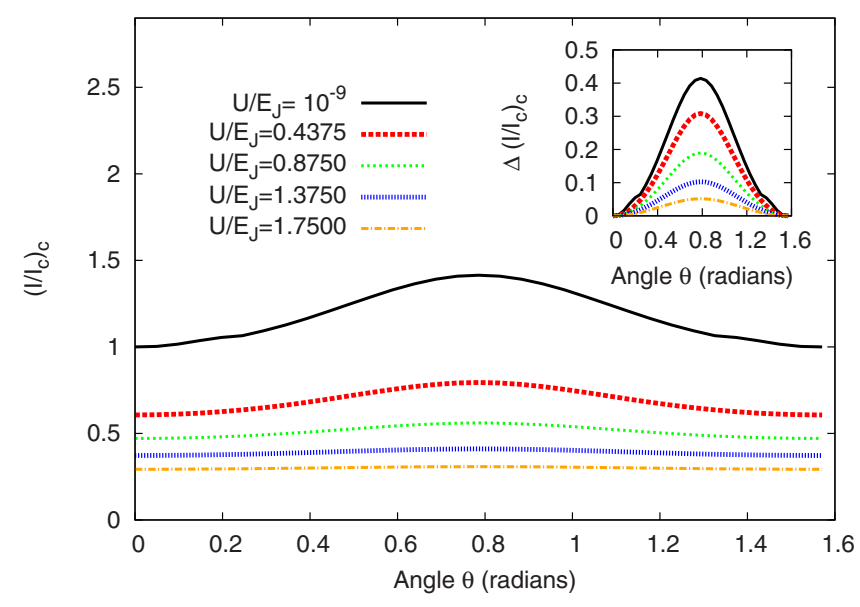

FIG. 6. (Color online) The dependence of $\left(I / I_{c}\right)_{c}$, representing the critical value of the ratio $I / I_{c}$, on bias angle $\theta$, for several values of $U / E_{J}$ The inset shows the scaled change $\Delta\left(I / I_{c}\right)_{c}$, in $\left(I / I_{c}\right)_{c}$, defined as $\Delta\left(I / I_{c}\right)_{c}=\left[\left(I / I_{c}\right)_{c}(\theta)-\left(I / I_{c}\right)_{c}(\theta=0)\right] /\left(I / I_{c}\right)_{c}(\theta=0)$, plotted as a function of $\theta$ for the same values of $U / E_{J}$. Here $I$ refers to the entire current across each plaquette, as discussed in the text.

energies. For a (3D) simple cubic lattice, the general behavior of the superconductor-to-insulator transition is similar to that of the square lattice. At a given $I / I_{c}$, however, the critical values of $U / E_{J}$ are somewhat lower than in the square lattice; the maximum value of this ratio at $I / I_{c}=0$ is 2.735 . The phase diagram resulting from our variational treatment is shown in Fig. 7, where we plot $\left(U / E_{J}\right)_{c}$ as a function of $I / I_{c}$. Here the injected current along each of the cube axes is $I / \sqrt{3}$ per junction so that the current is effectively applied along the body diagonal. The points on the curve in Fig. 7 were calculated similarly to the $2 \mathrm{D}$ case. For a given value of $I / I_{c}$ the program stepped through increasing values of $U / E_{J}$, checking for the highest value at which the free energy in Eq. (41) still had a local minimum. Once the initial estimates of $\left(U / E_{J}\right)_{c}$ and $\left(E_{t} / E_{J}\right)_{c}$ were obtained, the process was repeated several times within progressively narrower parameter spaces, as in the 2D case.

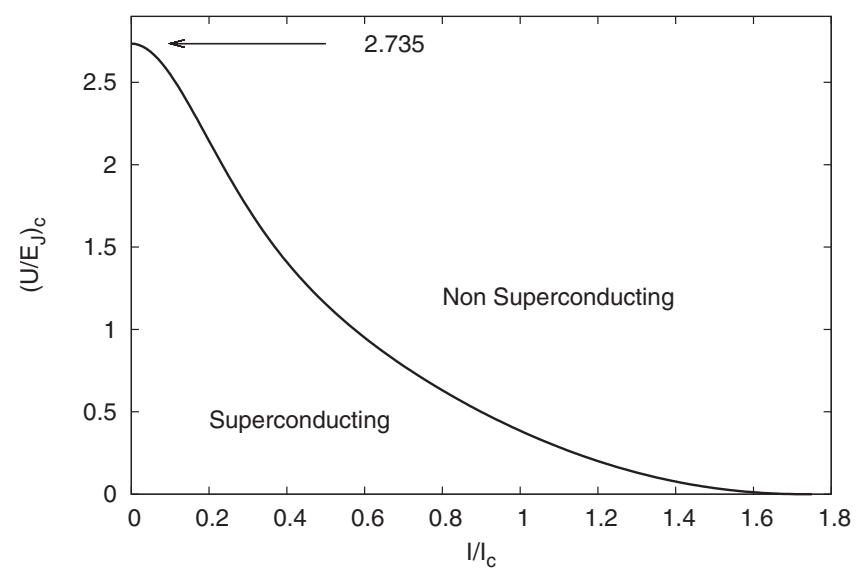

FIG. 7. Critical value of the ratio $a=U / E_{J}$ as a function of applied current $I / I_{c}$ for a cubic lattice. Here the current is taken to be along the body diagonal; the applied current per bond along each axis is $I / \sqrt{3}$. 


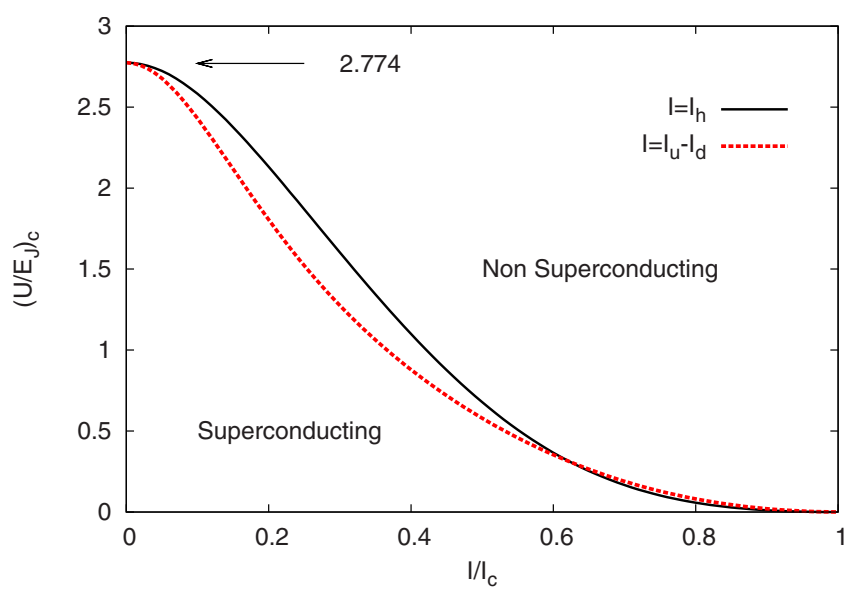

FIG. 8. (Color online) Critical values of the ratio $U / E_{J}$ as a function of $I / I_{c}$ for a triangular lattice and the two types of bias current considered. For the solid line labeled " $I=I_{h}$ " the applied bias current is applied parallel along the $x$ axis, so $I_{h}=I$ and $I_{u}=I_{d}$ $=0$. For the dashed line labeled " $I=I_{u}-I_{d}$ " the applied bias current is applied along the $y$ axis of Fig. 2, so that $I_{u}=-I_{d}=\sqrt{3} I / 2$ and $I_{h}$ $=0$ in the notation of Fig. 2. In this Figure, $I$ denotes the current along a given bond.

We have also carried out analogous calculations of $\left(U / E_{J}\right)_{c}$ for a triangular lattice. The results are qualitatively similar to the other calculations discussed above, though the applied currents must be differently parametrized, as discussed in Sec. II E. We have numerically considered only two simple cases. First, we have examined a current applied parallel to one edge of the triangles, e.g., the $x$ axis in Fig. 2. In this case, we use $I_{h}=I, I_{d}=I_{u}=0$ (in the notation of Sec. II E) in Eq. (54). In the second case, we consider an injected current applied along the $y$ axis of Fig. 2. In this case, we assume, in the notation of Sec. II E, that $I_{u}=-I_{d}=\sqrt{3} I / 2$. The resulting variational phase diagrams for these two cases are shown in Fig. 8. The curves in Fig. 8 were calculated in exactly the same manner described for the $2 \mathrm{D}$ square and $3 \mathrm{D}$ cubic lattices.

For all the lattices we have considered, the critical value, $a_{c}=\left(U / E_{J}\right)_{c}$ is that value of $a$ at which the metastable minimum in $F_{\text {var }}$ disappears when $F_{\text {var }}\left(E_{t}, a\right)$ is plotted against $E_{t}$. Although $E_{t}$ does not have direct physical significance, the corresponding value of $\left\langle\left(\phi_{i}-\phi_{j}\right)^{2}\right\rangle_{0}$ for adjacent superconducting islands does since it is a measure of the zeropoint quantum fluctuations. Within the variational approximation, it can be shown that

$$
\left\langle\left(\phi_{i}-\phi_{j}\right)^{2}\right\rangle_{0}=\xi \sqrt{\frac{U E_{J}}{E_{t}^{2}}}+\left(\frac{E_{J} I_{i j}}{E_{t} I_{c}}\right)^{2},
$$

where $I_{i j}$ is the current between the $i$ th and $j$ th island, and $\xi$ is a geometry-dependent numerical prefactor. We have, in fact, calculated $\xi$ for each geometry earlier in this paper, where it was labeled $\alpha, \beta$, and $\gamma$ for the square, cubic, and triangular cases, respectively. The method of obtaining critical values needed to compute these phase differences in Eq. (59) has already been discussed for previous figures. In Fig. 9, we show these squared phase differences at the critical

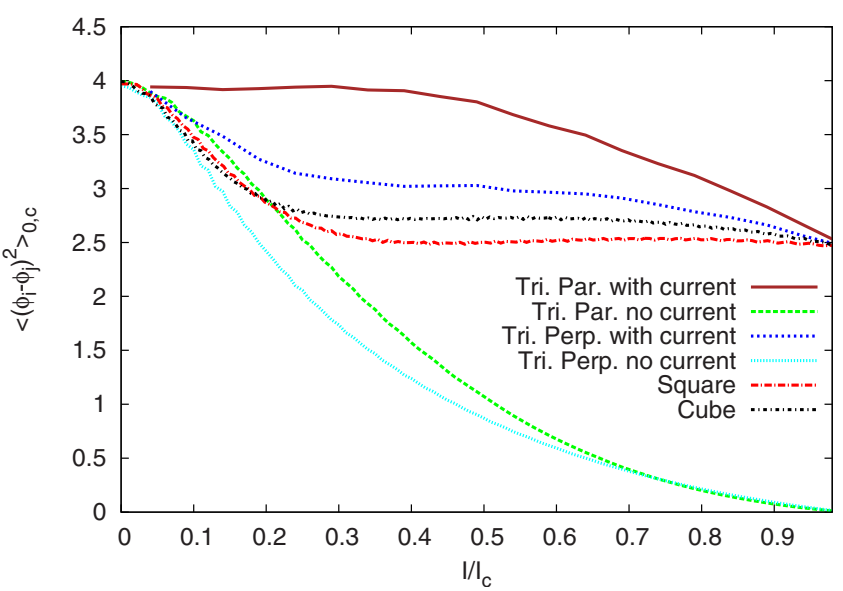

FIG. 9. (Color online) Variationally calculated critical meansquare phase difference $\left\langle\left(\phi_{i}-\phi_{j}\right)^{2}\right\rangle_{0, c}$ for different nearest-neighbor bonds, plotted as a function of applied current $I / I_{c}$ for the various geometries. For the square or cubic lattices with current applied along the diagonal or body diagonal, all such mean-square phase differences are equal; they are denoted "Square" or "Cube." "Tri.Par. with current" and "Tri.Par. no current" both refer to the triangular lattice with current applied along the $x$ axis but for bonds parallel and nonparallel to the $x$ axis, respectively. "Tri.Perp with current" and "Tri.Perp no current" refer to the triangular lattice with current perpendicular to the $x$ axis, and bonds perpendicular and parallel to the $x$ axis, respectively. In this figure $I$ refers to the current along a single bond.

values of $E_{t} / E_{J}$ for all the geometries considered, as a function of $I / I_{c}$.

Several aspects of this critical phase difference behavior are consistent with expectations. Consider, for example these critical values for each array as $I$ approaches $I_{c}$. One could roughly guess the magnitude of the critical phase difference by examining the initial Hamiltonian (1) to see where the metastable minima in the potential term vanish. Taking the derivative of Eq. (1) with respect to the phase difference $\left(\phi_{i}-\phi_{j}\right)$, and allowing $I$ to approach $I_{c}$, one obtains for any specific pair of adjacent islands $\sin \left(\phi_{i}-\phi_{j}\right)-I_{i j} / I_{c}=0$, or $\phi_{i}$ $-\phi_{j}=\arcsin (1)=\pi / 2$. This would correspond to an expectation value $\left\langle\left(\phi_{i}-\phi_{j}\right)^{2}\right\rangle_{0, c}=(\pi / 2)^{2}=2.467$. By comparison, the results shown in Fig. 9 give $\left\langle\left(\phi_{i}-\phi_{j}\right)^{2}\right\rangle_{0, c} \sim 2.476$ as $I \rightarrow I_{c}$ for several classes of bonds in different lattices. The only curves that do not approach this value correspond to neighbors between which no current is flowing. In the present work, such bonds are found only in the triangular lattice. Applying the argument above to a pair of islands with no current between them would yield an expected critical phase difference of $\left\langle\left(\phi_{i}-\phi_{j}\right)^{2}\right\rangle_{0, c}=\arcsin (0)^{2}=0$. In Fig. 9, these curves do indeed approach zero as the current (between other neighbors) approaches the critical current for a single junction.

Finally, we have calculated the finite- $T$ phase diagram for a square lattice with current injected along the diagonal. The method used to obtain critical values at nonzero temperature is identical to the numerical method described above for zero-temperature cases. The scanning of the temperaturedependent free energy in Eq. (58) is more computationally demanding, as it contains nontrivial, temperature-dependent 


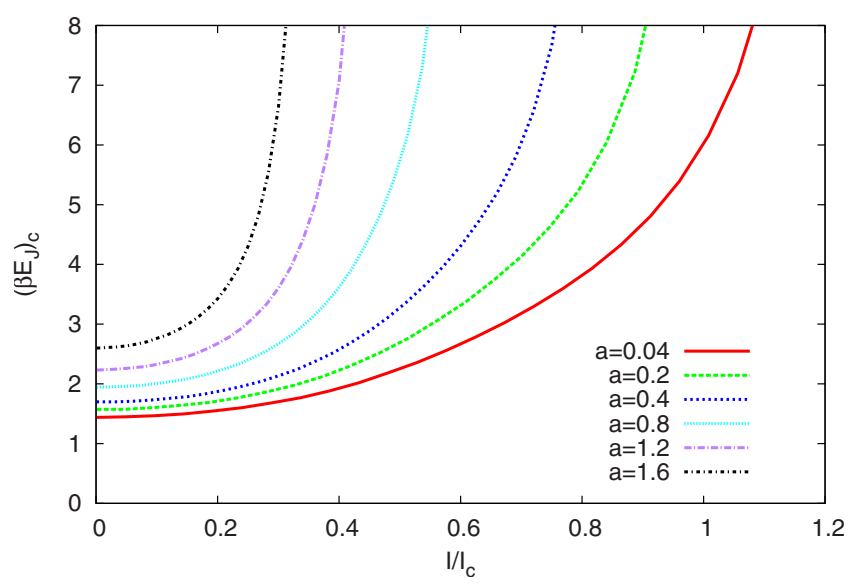

FIG. 10. (Color online) The critical values $\beta E_{J}$, denoted $\left(\beta E_{J}\right)_{c}$, plotted as functions of $I / I_{c}$ for various values of the parameter $a$ $=U / E_{J}$ for a square lattice. The applied currents along the $x$ and $y$ axes are each $I / \sqrt{2}$ and $\beta=1 / k_{B} T$.

integrals $D_{i j}(T)$ from Eq. (57). In Fig. 10 we show the calculated critical values of $E_{J} /\left(k_{B} T\right)$ as a function of $I / I_{c}$ for several values of $U / E_{J}$. These plots show, as expected, that the superconducting state occupies a progressively smaller part of the phase diagram, as $T$ increases.

\section{CONCLUSIONS}

In this paper, we have developed a simple variational approximation for the phase diagram of an array of small Josephson junctions in an applied dc current. We use this approach to calculate the superconducting/nonsuperconducting phase boundary at $T=0$, as a function of the magnitude and direction of the applied current. We also generalize this approach to finite temperatures.

It would be of much interest if this predicted phase diagram could be confirmed by a more exact study of this model. At zero current, our model, in $d$ dimensions, is believed to be in the same universality class as the $(d+1)$-dimensional XY model. ${ }^{51}$ Thus, in $d=2$, at $I=0$ and $T=0$, the model undergoes a continuous phase transition from a superconductor to an insulator as the parameter $U / E_{J}$ is increased with critical behavior characteristic of a $(d+1)$-dimensional XY model near the phase transition. This behavior can be verified by quantum Monte Carlo simulations, as has been shown by several groups. ${ }^{52}$ It would be of much interest if the same kind of calculations could be generalized to treat our model at finite $I$.

A comparison between the present results and experiment would also be of great interest. To our knowledge, no experi- ments have been carried out which probe the regime we are studying variationally. To carry out such an experiment would likely be challenging experimentally because any given realization of an array would have only a single value of the parameter $U / E_{J}$. Ideally, one would like to produce an array with a value of $U / E_{J}$ such that the array would be superconducting at $I=0$ but nonsuperconducting at larger $I$. Then, by sweeping the applied current through its critical value, one would be able to observe a superconductor/ nonsuperconductor transition. To obtain the full phase diagram, one would need to study experimentally several arrays, each well-ordered and having a different value of the parameter $U / E_{J}$. Ideally, one should carry out this current sweep for several directions of the applied current. The closest experimental study of which we are familiar ${ }^{42}$ involves an array of superconducting-normal-superconducting junctions in a transverse magnetic field (as well as an applied current). In our case, the desired experiment would involve an SIS array without a transverse magnetic field. It seems possible, if challenging, to carry out such experiments for SIS arrays.

In interpreting these proposed experiments, one would need to take into account unavoidable difficulties such as thermal fluctuations and junction-to-junction variations in the junction critical current and capacitance. Thermal fluctuations can be treated within our variational approximation, as shown in Fig. 10. It should be possible to carry out experiments at low enough temperatures to minimize such fluctuations and observe the predicted phase diagram. In any case, such fluctuations can be accounted for approximately, using the variational approach. As for disorder, we believe that this would have primarily a quantitative effect on the phase transition (i.e., would shift the transition current) without causing the phase transition to disappear altogether. It may be difficult to verify this hypothesis about the effects of disorder without additional calculations, however. If the junction parameters can be controlled to within perhaps $\pm 20 \%$, we speculate that the corresponding disorder would have little effect on the parameters of the phase transition. We conclude, therefore, that the phase transition proposed in this paper should be detectable in realistic Josephson junctions with experimentally achievable parameters.

\section{ACKNOWLEDGMENTS}

This work was supported by NSF under Grant No. DMR04-13395 and by the Center for Emerging Materials at Ohio State University, an NSF MRSEC (Grant No. DMR0820414).

\footnotetext{
${ }^{1}$ For a recent review, see, e.g., C. J. Lobb and R. S. Newrock, Solid State Physics: Advances in Research and Applications (Academic Press, San Diego, 2000), Vol. 54, pp. 263-512.

${ }^{2}$ R. Fazio and H. van der Zant, Phys. Rep. 355, 235 (2001).

${ }^{3}$ L. B. Ioffe, M. V. Feigel'man, A. Ioselevich, D. Ivanov, M.
}

Troyer, and G. Blatter, Nature (London) 415, 503 (2002).

${ }^{4}$ G. G. Batrouni and R. T. Scalettar, Phys. Rev. Lett. 84, 1599 (2000).

${ }^{5}$ T. D. Kühner, S. R. White, and H. Monien, Phys. Rev. B 61, 12474 (2000). 
${ }^{6}$ A. Romito, R. Fazio, and C. Bruder, Phys. Rev. B 71, 100501 (2005).

${ }^{7}$ A. Kapitulnik, N. Mason, S. A. Kivelson, and S. Chakravarty, Phys. Rev. B 63, 125322 (2001).

${ }^{8}$ B. Douçot, M. V. Feigel'man, and L. B. Ioffe, Phys. Rev. Lett. 90, 107003 (2003).

${ }^{9}$ A. Kampf and G. Schön, Phys. Rev. B 36, 3651 (1987).

${ }^{10}$ S. Teitel and C. Jayaprakash, Phys. Rev. Lett. 51, 1999 (1983).

${ }^{11}$ B. J. Kim, P. Minnhagen, and P. Olsson, Phys. Rev. B 59, 11506 (1999).

${ }^{12}$ V. I. Marconi and D. Dominguez, Phys. Rev. B 63, 174509 (2001).

${ }^{13}$ E. Trías, J. J. Mazo, F. Falo, and T. P. Orlando, Phys. Rev. E 61, 2257 (2000)

${ }^{14}$ B. G. Orr, H. M. Jaeger, A. M. Goldman, and C. G. Kuper, Phys. Rev. Lett. 56, 378 (1986).

${ }^{15}$ H. S. J. van der Zant, W. J. Elion, L. J. Geerligs, and J. E. Mooij, Phys. Rev. B 54, 10081 (1996).

${ }^{16}$ Y. Takahide, R. Yagi, A. Kanda, Y. Ootuka, and S. I. Kobayashi, Phys. Rev. Lett. 85, 1974 (2000).

${ }^{17}$ J. Holzer, R. S. Newrock, C. J. Lobb, T. Aouaroun, and S. T. Herbert, Phys. Rev. B 63, 184508 (2001).

${ }^{18}$ A. J. Rimberg, T. R. Ho, C. Kurdak, J. Clarke, K. L. Campman, and A. C. Gossard, Phys. Rev. Lett. 78, 2632 (1997).

${ }^{19}$ M. Greiner, O. Mandel, T. Esslinger, T. W. Hänsch, and I. Bloch, Nature (London) 415, 39 (2002).

${ }^{20}$ F. S. Cataliotti, S. Burger, C. Fort, P. Maddaloni, T. Minardi, A. Trombettoni, A. Smerzi, and M. Inguscio, Science 293, 843 (2001).

${ }^{21}$ D. B. Haviland and P. Delsing, Phys. Rev. B 54, R6857 (1996).

${ }^{22}$ K. H. Lee and D. Stroud, Phys. Rev. B 43, 5280 (1991).

${ }^{23}$ P. Barbara, B. Vasilic, A. B. Cawthorne, S. V. Shitov, and C. J. Lobb, Advances in Superconductivity XII (Springer-Verlag, Tokyo, 2000), p. 1102.

${ }^{24}$ J. Oppenländer, C. Häussler, and N. Schopohl, Phys. Rev. B 63, 024511 (2000).

${ }^{25}$ G. Deutscher, Y. Imry, and L. Gunther, Phys. Rev. B 10, 4598 (1974).

${ }^{26}$ E. Simánek, Inhomogeneous Superconductors: Granular and Quantum Effects (Oxford University Press, New York, 1994).

${ }^{27}$ Y. Nakamura, Yu. A. Pashkin, and J. S. Tsai, Nature (London) 398, 786 (1999).

${ }^{28}$ See, e.g., S. Gladchenko, D. Olaya, E. Dupont-Ferrier, B. Douçot, L. B. Ioffe, and M. E. Gershenson, Nat. Phys. 5, 48 (2009).

${ }^{29}$ This form of the Josephson coupling term is not valid in all cases; in particular, the coupling between SNS junctions may sometimes have a noncosinusoidal dependence on the phase. See, for example, A. A. Golubov, M. Yu. Kupriyanov, and E. Il'ichev, Rev. Mod. Phys. 76, 411 (2004).

${ }^{30}$ V. Berezinskii, Zh. Eksp. Teor. Fiz. 59, 907 (1970) [Sov. Phys. JETP 32, 493 (1971)].

${ }^{31}$ J. M. Kosterlitz and D. J. Thouless, J. Phys. C 6, 1181 (1973).

${ }^{32}$ S. L. Sondhi, S. M. Girvin, J. P. Carini, and D. Shahar, Rev. Mod. Phys. 69, 315 (1997).

${ }^{33}$ V. U. Ambegaokar and B. I. Halperin, Phys. Rev. Lett. 22, 1364 (1969).

${ }^{34}$ N. D. Mermin and H. Wagner, Phys. Rev. Lett. 17, 1133 (1966).

${ }^{35}$ M. S. Rzchowski, S. P. Benz, M. Tinkham, and C. J. Lobb, Phys. Rev. B 42, 2041 (1990).

${ }^{36}$ C. J. Lobb, D. W. Abraham, and M. Tinkham, Phys. Rev. B 27, 150 (1983)

${ }^{37}$ S. P. Benz, M. S. Rzchowski, M. Tinkham, and C. J. Lobb, Phys. Rev. B 42, 6165 (1990).

${ }^{38}$ H. S. J. van der Zant, F. C. Fritschy, T. P. Orlando, and J. E. Mooij, Phys. Rev. B 47, 295 (1993).

${ }^{39}$ C. Reichhardt and C. J. Olson, Phys. Rev. B 65, 174523 (2002).

${ }^{40}$ C. Reichhardt, C. J. Olson, and F. Nori, Phys. Rev. B 58, 6534 (1998).

${ }^{41}$ K. D. Fisher, D. Stroud, and L. Janin, Phys. Rev. B 60, 15371 (1999).

${ }^{42}$ I. Elsayed, K. H. Sarwa B. Tan, K. A. Parendo, and A. M. Goldman, Phys. Rev. B 80, 052502 (2009).

${ }^{43}$ K. Wiesenfeld, P. Colet, and S. H. Strogatz, Phys. Rev. Lett. 76, 404 (1996).

${ }^{44}$ See, e.g., S. Ryu, M. Hellerqvist, S. Doniach, A. Kapitulnik, and D. Stroud, Phys. Rev. Lett. 77, 5114 (1996); P. Le Doussal and T. Giamarchi, Phys. Rev. B 57, 11356 (1998); L. Balents, M. C. Marchetti, and L. Radzihovsky, ibid. 57, 7705 (1998); C. Reichhardt, G. T. Zimanyi, and N. Grönbech-Jensen, ibid. 64, 014501 (2001); D. Domínguez, Phys. Rev. Lett. 82, 181 (1999).

${ }^{45}$ D. M. Wood and D. Stroud, Phys. Rev. B 25, 1600 (1982).

${ }^{46}$ J. W. Gibbs, The Collected Works of J. Willard Gibbs (Yale University Press, New Haven, 1948), Vol. II, Chap. XI, p. $129 \mathrm{ff}$.

${ }^{47}$ P. Choquard, The Anharmonic Crystal (W. A. Benjamin, New York, 1967), p. 184f.

${ }^{48}$ K. Kim and D. Stroud, Phys. Rev. B 78, 174517 (2008).

${ }^{49}$ Y.-H. Li and S. Teitel, Phys. Rev. B 40, 9122 (1989).

${ }^{50}$ T. K. Kopeć and J. V. José, Phys. Rev. B 60, 7473 (1999).

${ }^{51}$ M. P. A. Fisher, P. B. Weichman, G. Grinstein, and D. S. Fisher, Phys. Rev. B 40, 546 (1989).

${ }^{52}$ See, e.g., M. C. Cha, M. P. A. Fisher, S. M. Girvin, M. Wallin, and A. P. Young, Phys. Rev. B 44, 6883 (1991). 